Research Article

\title{
Effect of Coptis chinensis on Biofilm Formation and Antibiotic Susceptibility in Mycobacterium abscessus
}

\author{
Cheng-Yin Tseng, ${ }^{1,2}$ Mao-Feng Sun, ${ }^{3,4}$ Tsai-Chung Li, ${ }^{5}$ and Ching-Ting Lin ${ }^{6}{ }^{6}$ \\ ${ }^{1}$ Graduate Institute of Chinese Medicine, School of Chinese Medicine, China Medical University, 91 Hsueh-Shih Rd, \\ Taichung City 40402, Taiwan \\ ${ }^{2}$ Section of Infectious Disease, Hsinchu Mackay Memorial Hospital, 690, Sec. 2, Guang-fu Rd., East Dist, \\ Hsinchu City 30071, Taiwan \\ ${ }^{3}$ Department of Chinese Medicine, China Medical University Hospital, 2 Yu-de Rd, North Dist., Taichung City 40447, Taiwan \\ ${ }^{4}$ School of Chinese Medicine, China Medical University, 91 Hsueh-Shih Rd, Taichung City 40402, Taiwan \\ ${ }^{5}$ Department of Public Health, College of Public Health, China Medical University, 91 Hsueh-Shih Rd, \\ Taichung City 40402, Taiwan \\ ${ }^{6}$ School of Chinese Medicine, China Medical University, 91 Hsueh-Shih Rd, Taichung City 40402, Taiwan
}

Correspondence should be addressed to Ching-Ting Lin; gingting@mail.cmu.edu.tw

Received 17 June 2020; Revised 23 October 2020; Accepted 29 October 2020; Published 10 November 2020

Academic Editor: Rômulo Dias Novaes

Copyright (c) 2020 Cheng-Yin Tseng et al. This is an open access article distributed under the Creative Commons Attribution License, which permits unrestricted use, distribution, and reproduction in any medium, provided the original work is properly cited.

\begin{abstract}
Mycobacterium abscessus infections are notoriously difficult to be treated and newer treatment options are required. Coptis chinensis (C. chinensis) and its main compound berberine are frequently used to treat bacterial and viral infections. In this study, the susceptibility of M. abscessus to C. chinensis extract and berberine was assessed by minimal inhibitory concentration (MIC) and minimal bactericidal concentration $(\mathrm{MBC})$ evaluation. The effects of $C$. chinensis and berberine on biofilm formation and antibiotic susceptibility in M. abscessus were observed. C. chinensis at concentrations of MIC $(1.5 \mathrm{mg} / \mathrm{mL})$ and $2 \times \mathrm{MIC}(3.0 \mathrm{mg} /$ $\mathrm{mL})$ and berberine at $1 / 2 \times \mathrm{MIC}(0.125 \mathrm{mg} / \mathrm{mL})$ demonstrated a strong inhibition of biofilm formation. Concentration of C. chinensis at $1 / 2 \times$ MIC resulted in a significant reduction in MICs of trimethoprim/sulfamethoxazole (TMP/SXT), clarithromycin (CLA), and linezolid (LZD). Similarly, $1 / 2 \times$ MIC berberine had a significant effect on the MIC reductions of nine antibiotics including TMP/SXT, CLA, and LZD. Notably, the resistance level MIC of LZD against M. abscessus was reversed to a susceptible level by treatment with either $C$. chinensis or berberine. Therefore, $C$. chinensis and berberine have the potential to produce a synergistic antimycobacterial effect, reduce biofilm formation, and decrease antibacterial resistance to LZD in M. abscessus.
\end{abstract}

\section{Introduction}

Nontuberculous mycobacteria (NTM), also known as environmental mycobacteria, are ubiquitous in a variety of natural habitats (wet soil, marshlands, streams, rivers, and estuaries) and man-made habitats (canals, pastures, and drinking water distribution systems). To date, more than 172 NTM species have been identified [1-3]. Although most species are nonpathogenic, Thomson et al. found pathogenic NTM species in large urban water distribution systems in Australia [4]. Human NTM diseases (e.g., pulmonary diseases, lymphadenitis, skin and soft tissue infections, and disseminated diseases) are believed to result from environmental exposure, trauma, and surgical or cosmetic procedures [5]. In 2019, Hung et al. reported a cluster of patients with postoperative M. abscessus endophthalmitis [6]. Wentworth et al. reported a threefold increase in the incidence of cutaneous NTM infections from 1980 to 2009 [7]. A study in Taiwan reported an average of 12.5 cases of NTM skin and soft tissue infection per year, which was an increase from previous years [8]. The prevalence of NTM infections in immunocompromised patients, including 
AIDS patients and transplant recipients, is growing worldwide [2, 9-11]. The number of NTM disease-related deaths is also rising, with higher mortality rates seen among adults over the age of 55 years and those with chronic obstructive pulmonary disease, bronchiectasis, HIV, interstitial lung diseases, and/or tobacco use [12].

While there is a wide range of treatment strategies available with multidrug antibiotic therapy being the current treatment of choice $[13,14]$, the results of antibiotic treatment are often discouraging [14]. Patients require a prolonged antibiotic course, which often results in side effects, and/or acquired antibiotic resistance. Suggested treatment is antibiotic therapy lasting 9-18 months, or even up to 24 months [15]. Interestingly, M. abscessus infections are widely seen hard to be treated despite the use of antibiotics. In a study of antibiotic therapy for pulmonary $M$. abscessus infections over a median duration of 12 months (range 9-18 months) by Novosad et al., $62 \%$ of patients experienced side effects that resulted in the need to adjust the therapeutic regimen [14]. Adjuvant or alternative therapies may be required, and a response to antibiotic side effects is needed. Adding Chinese herbal medicine (CHM) to antibiotic treatments may provide an effective solution for treating antibiotic side effects.

$\mathrm{CHM}$ has been used to treat infectious diseases since ancient times in China. Over thousands of years, many mechanisms have been proposed and countless reports of CHM antimicrobial therapy have been published in scientific publications and textbooks, such as the classic Chinese medical monographs "Treatise on Febrile Diseases (Shāng Hán Lùn)" and "Systematic Differentiation of Warm Diseases (Wèn Bing Tiáo Biàn).” In recent decades, a large number of studies have reported on the anti-inflammatory and antimicrobial effects of heat-clearing Chinese herbs (HCCHs) [16-18]. Currently, HCCHs and CHM formulations are frequently used as monotherapy or combined therapy components to treat common infectious diseases such as pulmonary tuberculosis, methicillin-resistant Staphylococcus aureus (MRSA) infection, and sepsis [19-21]. CHM formulations with bacteriostatic activity may be useful in treating $M$. abscessus infections.

Coptis chinensis (C. chinensis; Huang Lian) is a traditional CHM frequently used to treat bacterial and viral infections. It has proven efficacy against many microorganisms including Staphylococcus spp. [18]. In 2006, Lin et al. found that Coptidis Rhizoma (the dried rhizome of C. chinensis Franch) provided $100 \%$ in vitro inhibitory effects on S. aureus and Escherichia coli [22]. Of note, C. chinensis was reported to have an effect comparable to that of the commercial antibiotic vancomycin against coagulase-negative Staphylococcus [23]. Recently, C. chinensis was found to produce significant antimicrobial activity against a variety of microorganisms including Gram-positive and Gram-negative bacteria [16]. Over 120 chemical components have been isolated from $C$. chinensis, with alkaloids as the main active ingredients and berberine as the most representative component [24]. Berberine's broadspectrum antibacterial effect is similar to that of $C$. chinensis, with effects manifesting proportional to concentrations. Higher concentrations of berberine are reported to kill bacteria more quickly [25]. In this era of increasing antibiotic resistance, the potential benefits of using $C$. chinensis and berberine in the treatment of $M$. abscessus infections warrant investigation. The aims of this study were to examine in vitro the antibacterial effect of $C$. chinensis and berberine (its major constituent) in treating $M$. abscessus infections. The researchers studied the potential of this herb to inhibit biofilm formation and amplify the activity of antibiotics in managing M. abscessus infections.

\section{Materials and Methods}

For this study, the M. abscessus strain, which was purchased from the College of American Pathologists for qualification of Medical Technologist, from the stock of Medical Laboratory of the Tamshui Branch of Mackay Memorial Hospital in Taiwan, was chosen to represent rapidly growing mycobacterium (RGM). An incubation broth (7H9) and commercially available antimycobacterial susceptibility test kit (Sensititre ${ }^{\mathrm{TM}}$ RAPMYCOI, Trek Sensititre, ThermoFisher Scientific) were used to test the susceptibility of the RGM to a panel of 15 antibiotics which is recommended by the Clinical and Laboratory Standards Institute (CLSI) [26]. For the purpose of comparing the actual inhibitory activities of pretreatment (antibiotic alone) and combination treatment (antibiotic plus C. chinensis or berberine), the Sensititre ${ }^{\mathrm{TM}}$ RAPMYCOI was further used to test the synergistic antibacterial effects of $C$. chinensis and berberine. Based on our review of Chinese Medicine books, research reports, and discussions with traditional Chinese medicine physicians, we selected the CHM C. chinensis for this study, an herb containing $6.19 \%$ berberine. In order to overcome the possible issues, such as sample preparation or different batch of raw material, and to make the results more reliable, a pharmacy type of concentrated $C$. chinensis paste which was approved for clinical use by the Ministry of Health and Welfare, Taiwan (provided by Kaiser Pharmaceutical Co., Ltd; product: 8010) was used. The herbal materials provided by Kaiser Pharmaceutical conformed to the standards of Taiwan Herbal Pharmacopeia 2. The procedures complied with the drugs registration license approved by the Taiwan Food and Drug Administration, in which the extraction of the raw material is performed exclusively using water. Additionally, the concentrations of commercially available berberine and berberine- $\mathrm{HCl}$ obtained from the products of C. chinensis were tested by Kaiser Pharmaceutical by HPLC following the protocol listed in Taiwan Herbal Pharmacopeia 2 . The antimicrobial activities of commercially available berberine and berberine- $\mathrm{HCl}$ were identical as examined with its antibacterial activities against M. abscessus. Thus, the erstwhile described berberine and berberine- $\mathrm{HCl}$ were purchased to enable a detailed analysis of its potential to enhance antibacterial effects (ISO 9001: 2015 certification; http://www.chemfaces.com).

2.1. M. abscessus Susceptibility to C. chinensis and Berberine. C. chinensis was dissolved in $7 \mathrm{H} 9$ broth and then serially diluted twofold from $12.0 \mathrm{mg} / \mathrm{mL}$ for testing concentration 
from $12.0 \mathrm{mg} / \mathrm{mL}$ to $0.375 \mathrm{mg} / \mathrm{mL}$. The antibacterial effects of berberine against $M$. abscessus were determined in the same manner, from $2.0 \mathrm{mg} / \mathrm{mL}$ to $0.0625 \mathrm{mg} / \mathrm{mL}$. The antibacterial activity of $C$. chinensis and berberine was evaluated by counting the colony forming unit (CFU) of M. abscessus found after their administration. As the protocol of TREK Diagnostic Systems, M. abscessus suspension (optical $\mathrm{OD}_{600} \sim 0.2$ ) was then added to the indicated concentrations of $C$. chinensis and berberine- $\mathrm{HCl}$ to observe the bacterial growth at $30^{\circ} \mathrm{C}$ for 5 days [27]. Relative percentage of survival rate was expressed as CFU of viable bacteria present after treatment with the indicated concentrations of $C$. chinensis or berberine- $\mathrm{HCl}$ relative to the number of viable bacteria present at untreatment and then multiplied by 100 .

2.2. Effects of C. chinensis and Berberine on the Biofilm Formation of $M$. abscessus. To evaluate the prevention effect before biofilm development, serial indicated concentrations of $C$. chinensis and berberine- $\mathrm{HCl}$ were individually added to the glass test tubes in the same time of preparation of M. abscessus solution to calculate optical density differences. Optical densities before and after the addition of $C$. chinensis and berberine- $\mathrm{HCl}$ were measured and compared.

Biofilm formation was assessed by the ability of cells to adhere to the glass tubes, with some modification of the reported protocol [28]. For testing, the concentration of C. chinensis ranging between 3 and $0.375 \mathrm{mg} / \mathrm{mL}$, and the concentration of berberine ranged between 0.5 and $0.0625 \mathrm{mg} / \mathrm{mL}$. The glass test tube contained a $1: 10$ diluted aliquot of $M$. abscessus overnight culture, which was incubated in $7 \mathrm{H} 9$ broth with different concentrations of C. chinensis and berberine- $\mathrm{HCl}$ at $30^{\circ} \mathrm{C}$ for 14 days. After 14 days, nonadherent $M$. abscessus was triple washed with $3 \mathrm{~mL}$ PBS, and the adherent M. abscessus was stained with $3 \mathrm{~mL}$ of $0.1 \%$ safranin solution at room temperature for $30 \mathrm{~min}$. The glass tube was rinsed twice with deionized water to remove excess stain. Finally, the safranin stained biofilm was solubilized in $1 \mathrm{~mL}$ of $33 \%$ acetic acid, and then $100 \mu \mathrm{L}$ of the solution was removed to 96-well PVC plates (TPP 96 flat) for measuring the absorbance at $\lambda=492 \mathrm{~nm}$.

2.3. Effects ofC. chinensis and Berberine on Minimal Inhibitory Concentrations (MICs) of Antibiotics against M. abscessus. Mycobacterium abscessus was treated with 15 antibiotics, without or with C. chinensis and berberine-HCl. MICs in this study were determined using a commercially available antimycobacterial susceptibility test kit (Sensititre ${ }^{\mathrm{TM}}$ RAPMYCOI, Trek Sensititre, ThermoFisher Scientific) according to the manufacturer's instructions. Briefly, $50 \mu \mathrm{L}$ of the M. abscessus suspension, grown in $7 \mathrm{H} 9$ broth at $30^{\circ} \mathrm{C}$ for 5 days and adjusted to optical $\mathrm{OD}_{600} \sim 0.2$, was added to $11 \mathrm{ml}$ 7H9 broth alone or containing the indicated concentrations of C. chinensis ( $3 \mathrm{mg} / \mathrm{mL}$ to $0.375 \mathrm{mg} / \mathrm{mL}$ ) or berberine $-\mathrm{HCl}$ $(0.5 \mathrm{mg} / \mathrm{mL}$ to $0.0625 \mathrm{mg} / \mathrm{mL})$ and mixed well. Then, $100 \mu \mathrm{L}$ of the mixture was added per well of the kit's Sensititre ${ }^{\text {TM }}$ Myco RAPMYCOI AST Plate, with evaluated concentrations of 15 antibiotics as $(\mu \mathrm{g} / \mathrm{ml})$ trimethoprim/ sulfamethoxazole, 0.25/4.75-8/152; linezolid, 1-32; ciprofloxacin, 0.12-4; imipenem, 2-64; moxifloxacin, 0.25-8; cefepime, 1-32; cefoxitin, 4-128; amoxicillin/clavulanic acid, 2/1-64/32; amikacin, 1-64; ceftriaxone, 4-64; doxycycline, 0.12-16; minocycline, 1-8; tigecycline, 0.015-4; tobramycin, 1-16; and clarithromycin, $0.06-16$, for incubating at $30^{\circ} \mathrm{C}$ for 5 days. Finally, we observed the effect of $C$. chinensis and berberine- $\mathrm{HCl}$ on the MICs of 15 antibiotics against M. abscessus. Both the MICs of the antibiotics alone and the $\mathrm{MIC}$ of the antibiotics plus $C$. chinensis and berberine- $\mathrm{HCl}$ were measured at least three times.

2.4. Statistical Analysis. The CFU numbers of $M$. abscessus were individually calculated after the indicated concentrations of $\mathrm{C}$. chinensis and berberine- $\mathrm{HCl}$ were added. The results were expressed as relative percentages \pm standard deviations (SD). The effects on biofilm formation after C. chinensis and berberine- $\mathrm{HCl}$ treatment were also analyzed. The results were expressed as mean scores \pm SD. The Mann-Whitney Rank Sum Test was conducted to examine any differences between control and experimental groups with indicated concentrations. The level of statistical significance was set at $p<0.05$.

\section{Results}

3.1. Susceptibility of M. abscessus to C. chinensis and Berberine. For evaluation of the effects of $C$. chinensis on the growth of M. abscessus, the number of $M$. abscessus CFU formed in 7H9 medium with different concentrations of $C$. chinensis was calculated and compared to the number of $M$. abscessus colonies formed in $7 \mathrm{H} 9$ medium alone (the control group). The relative survival percentages of $M$. abscessus were $59.95 \pm 13.70 \%, \quad 34.56 \pm 6.58 \%, \quad 15.20 \pm 4.74 \%$, and $2.55 \pm 0.27 \%$ at concentrations of $0.375 \mathrm{mg} / \mathrm{mL}, 0.75 \mathrm{mg} / \mathrm{mL}$, $1.5 \mathrm{mg} / \mathrm{mL}$, and $3.0 \mathrm{mg} / \mathrm{mL}$, respectively (Figure $1(\mathrm{a})$ ). Therefore, the MIC value of C. chinensis was around $1.5 \mathrm{mg} /$ $\mathrm{mL}$ for $M$. abscessus. Additionally, we observed that the growth of $M$. abscessus approached zero at $C$. chinensis concentration of $6.0 \mathrm{mg} / \mathrm{mL}(0.31 \pm 0.34 \%)$. This implies that minimal bactericidal concentration (MBC) was 4 times higher than MIC of $C$. chinensis. The reductions in relative percentage of survival CFU of $M$. abscessus were statistically significant for $1 / 4 \times \mathrm{MIC}, 1 / 2 \times \mathrm{MIC}, \mathrm{MIC}, 2 \times \mathrm{MIC}$, and $4 \times \mathrm{MIC}$ following the addition of $C$. chinensis. Our findings revealed that $C$. chinensis has a conspicuous bacteriostatic effect on $M$. abscessus.

Berberine is the main active component of $C$. chinensis. To further observe the effects of berberine on $M$. abscessus growth, the CFU numbers of $M$. abscessus with different concentrations of berberine- $\mathrm{HCl}$ were calculated and compared to the numbers of $M$. abscessus grown in $7 \mathrm{H} 9$ medium alone. The relative survival percentages of M. abscessus were $69.45 \pm 9.68 \%, \quad 31.41 \pm 2.50 \%$, $16.27 \pm 4.72 \%$, and $0.57 \pm 0.62 \%$ at berberine- $\mathrm{HCl}$ concentrations of $0.0625 \mathrm{mg} / \mathrm{mL}, 0.125 \mathrm{mg} / \mathrm{mL}, 0.25 \mathrm{mg} / \mathrm{mL}$, and $0.5 \mathrm{mg} / \mathrm{mL}$, respectively (Figure 1(b)). The MIC value of berberine- $\mathrm{HCl}$ was around $0.25 \mathrm{mg} / \mathrm{mL}$ for $M$. abscessus. 


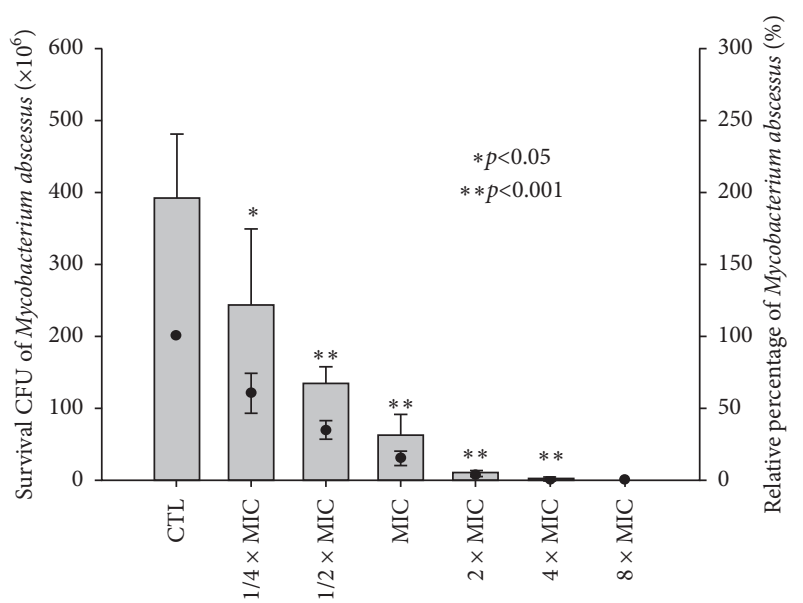

Coptis chinensis (minimal inhibitory concentration)

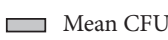

- Percentage

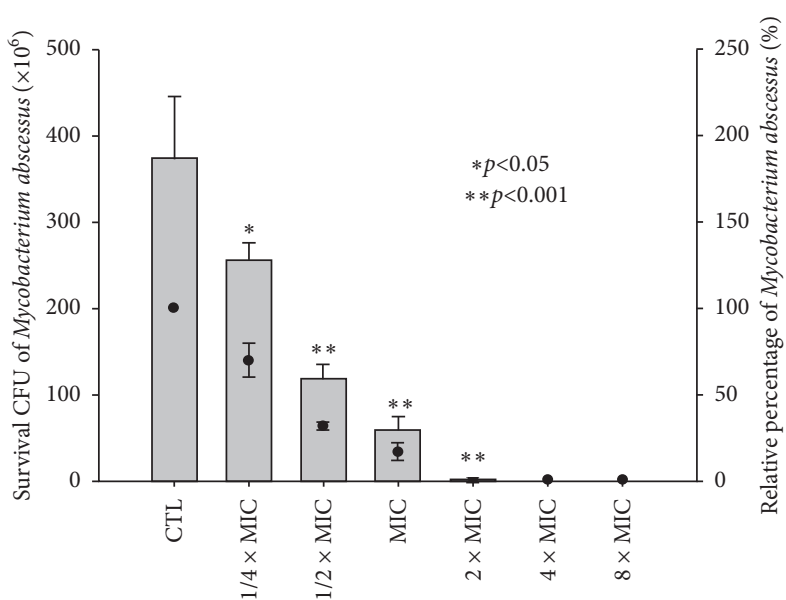

Berberine-HCI (minimal inhibitory concentration)

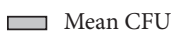

- Percentage

(a)

(b)

FIgURE 1: Susceptibility of M. abscessus to C. chinensis or berberine-HCl. The CFU formed of M. abscessus in 7H9 medium alone (the control group) and after treatment with the indicated concentrations of $C$. chinensis or berberine- $\mathrm{HCl}$ were counted by plating. Survival $\mathrm{CFU}$ and the relative percentage were expressed. As determined by the Mann-Whitney Rank Sum Test, statistically significant reductions (asterisk) in relative percentage of survival CFU of $M$. abscessus were observed for $1 / 4 \times$ MIC, $1 / 2 \times$ MIC, MIC, $2 \times$ MIC, and $4 \times$ MIC following the addition of Coptis chinensis (a) and 1/4 $\times$ MIC, $1 / 2 \times \mathrm{MIC}$, MIC, and $2 \times \mathrm{MIC}$ following the addition of berberine-HCl (b). CFU: colony forming unit. CTL: control. MIC: minimal inhibitory cncentration. Error bars indicate standard deviations. ${ }^{*} p<0.05$ and ${ }^{* *} p<0.001$.

Likewise, nearly no $M$. abscessus growth was observed with berberine- $\mathrm{HCl}$ concentrations in $0.5 \mathrm{mg} / \mathrm{mL}$ and in excess of $1.0 \mathrm{mg} / \mathrm{mL}$. The $\mathrm{MBC}$ of berberine- $\mathrm{HCl}$ was 2-4 times higher than MIC. As determined by the Mann-Whitney Rank Sum Test, the differences in relative percentage of survival CFU of $M$. abscessus between pretreatment and the addition of $1 / 4 \times \mathrm{MIC}, 1 / 2 \times \mathrm{MIC}, \mathrm{MIC}$, and $2 \times \mathrm{MIC}$ berberine were statistically significant. Our observations indicate that berberine-HCl also showed an obvious bacteriostatic effect against $M$. abscessus growth.

3.2. Effects of C. chinensis and Berberine on the Biofilm Formation of M. abscessus. The biofilm formation of M. abscessus on the glass tubes was reviewed and compared. The biofilm of M. abscessus declined after incubation with $C$. chinensis and berberine-HCl for 14 days. The effects manifested were proportional to the added concentrations. Higher concentrations of $C$. chinensis and berberine- $\mathrm{HCl}$ exhibited poor abilities of adherence. To analyze whether $C$. chinensis and berberine- $\mathrm{HCl}$ affect biofilm formation of $M$. abscessus, the biofilm was determined at $\mathrm{OD}_{492}$ by staining with safranin. As shown in Figure 2(a), the differences of optical density 492 were (mean \pm standard deviation) $0.872 \pm 0.0903,0.641 \pm 0.0530$, $0.344 \pm 0.0348,0.275 \pm 0.0767$, and $0.190 \pm 0.0312$ at control and concentrations of $1 / 4 \times \mathrm{MIC}, 1 / 2 \times \mathrm{MIC}, \mathrm{MIC}$, and $2 \times \mathrm{MIC}$ of $C$. chinensis, respectively. We found that the concentrations of C. chinensis at $1 / 4 \times \mathrm{MIC}, 1 / 2 \times \mathrm{MIC}, \mathrm{MIC}$, and $2 \times \mathrm{MIC}$ significantly decreased $M$. abscessus biofilm formation. These results indicate that the biofilm formation of $M$. abscessus could be inhibited by the concentration of $C$. chinensis in excess of $1 /$ $4 \times$ MIC. When indicated MICs of berberine- $\mathrm{HCl}$ were added
(Figure 2(b)), the differences of optical density 492 were (mean \pm standard deviation) $0.872 \pm 0.0903,0.723 \pm 0.0562$, $0.483 \pm 0.0580,0.137 \pm 0.00832$, and $0.110 \pm 0.00362$ at control and concentrations of $1 / 4 \times \mathrm{MIC}, 1 / 2 \times \mathrm{MIC}, \mathrm{MIC}$, and $2 \times \mathrm{MIC}$, respectively. We observed that biofilm formation of M. abscessus slightly decreased at $1 / 4 \times$ MIC and considerably decreased at $1 / 2 \times \mathrm{MIC}, \mathrm{MIC}$, and $2 \times \mathrm{MIC}$. These findings reveal that biofilm formation of $M$. abscessus could be consistently inhibited using berberine- $\mathrm{HCl}$ concentrations in excess of $1 / 2 \times$ MIC.

3.3. Effects of C. chinensis on the MICs of Antibiotics against M. abscessus. According to the reference MICs of antibiotics for M. abscessus, this study strain of M. abscessus was primarily resistant to most studied antibiotics except clarithromycin, tigecycline, and amikacin [26, 29]. To observe whether $C$. chinensis would affect the MICs of antibiotics against $M$. abscessus, combinations of antibiotics and C. chinensis were tested. The MICs of antibiotics alone and antibiotics combined with $C$. chinensis were then compared. The addition of $C$. chinensis in concentrations of $1 / 4 \times \mathrm{MIC}$, $1 / 2 \times$ MIC, MIC, and $2 \times$ MIC were tested to measure the effects on MICs of antibiotics. As shown in Table 1, the addition of $1 / 4 \times$ MIC $C$. chinensis provided a reduction of only MIC in linezolid. C. chinensis concentrations of $1 / 2 \times$ MIC and MIC resulted in significant synergistic effects measured for trimethoprim/sulfamethoxazole, clarithromycin, and linezolid. Importantly, the synergistic effect of $C$. chinensis on these three antibiotics is highly related to the concentration of $C$. chinensis. Furthermore, the concentration of $C$. chinensis at MIC could also reduce the MICs 
(i) M. abscessus was incubated in $7 \mathrm{H} 9$ broth with different concentrations of C. chinensis at $30^{\circ} \mathrm{C}$ for 14 days.

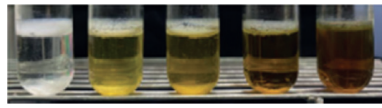

(ii) The C. chinensis solution was removed.

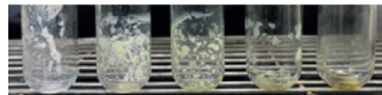

(iii) The adherent $M$. abscessus was stained with $3 \mathrm{~mL}$ of $0.1 \%$ safranin solution at room temperature for $30 \mathrm{~min}$.

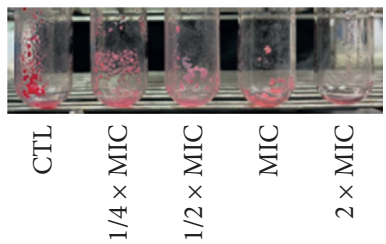

(iv) The safranin stained biofilm was measured the differences in $\lambda=492 \mathrm{~nm}$ at plates.
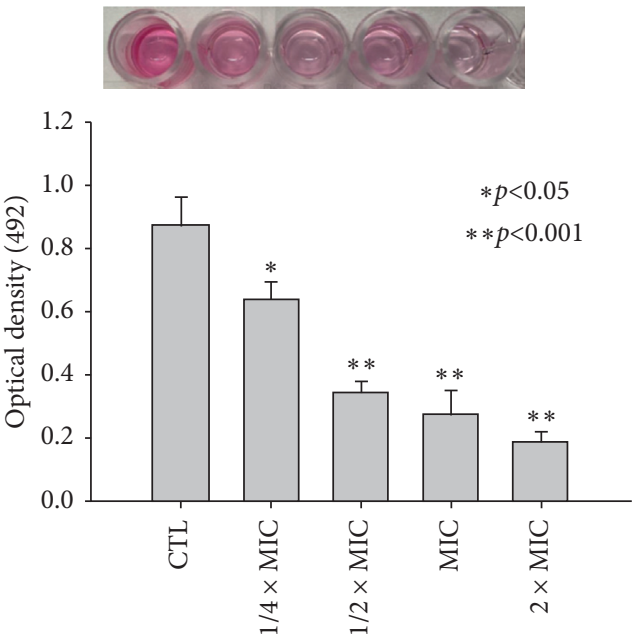

Coptis chinensis (minimal inhibitroy concentration)

(a) (i) M. abscessus was incubated in 7H9 broth with different concentrations of berberine-HCL at $30^{\circ} \mathrm{C}$ for 14 days.

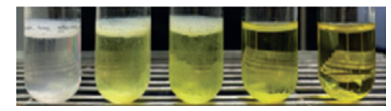

(ii) The berberine-HCL solution was removed.

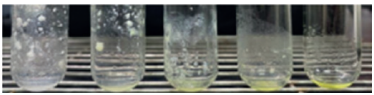

(iii) The adherent $M$. abscessus was stained with $3 \mathrm{~mL}$ of $0.1 \%$ safranin solution at room temperature for $30 \mathrm{~min}$.

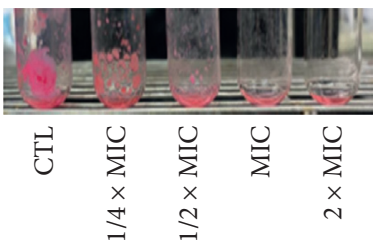

(iv) The safranin stained biofilm was measured the differences in $\lambda=492 \mathrm{~nm}$ at plates.

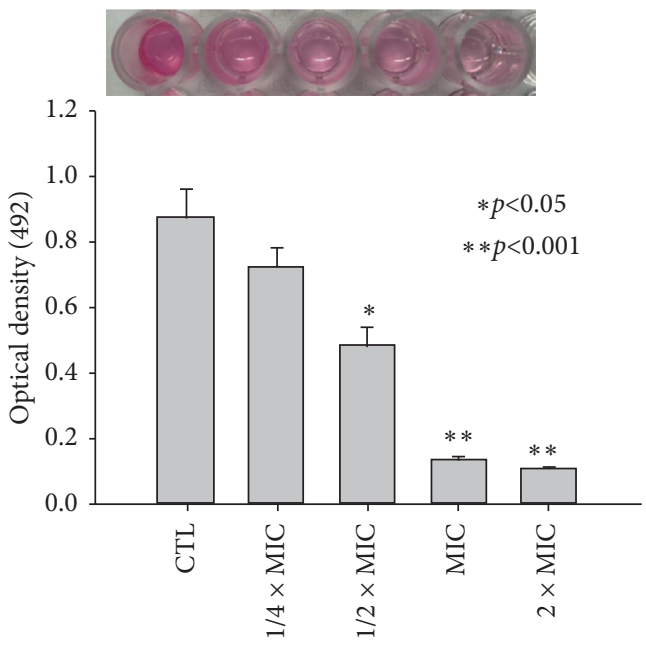

Berberine-HCI (minimal inhibitory concentration)

(b)

Figure 2: Comparative effects of indicated concentration of C. chinensis and berberine- $\mathrm{HCl}$ on the biofilm formation of $M$. abscessus. M. abscessus was grown in $7 \mathrm{H} 9$ broth (control) and under the concentrations of $1 / 4 \times \mathrm{MIC}, 1 / 2 \times \mathrm{MIC}$, MIC, and $2 \times$ MIC of C. chinensis (a) and berberine- $\mathrm{HCl}(\mathrm{b})$ at $30^{\circ} \mathrm{C}$ for 14 days. $M$. abscessus solution was incubated at $30^{\circ} \mathrm{C}$ for 14 days (i). After 14 days, nonadherent M. abscessus was removed (ii). The adherent $M$. abscessus was stained with $3 \mathrm{~mL}$ of $0.1 \%$ safranin solution at room temperature for $30 \mathrm{~min}$ (iii). The safranin stained biofilm was solubilized in acetic acid and then was removed to 96-well PVC plates for measuring the differences in optical density $492(\lambda=492 \mathrm{~nm})$ (iv). The photo represented biofilm solutions of control and indicated concentrations of $C$. chinensis and berberine- $\mathrm{HCl}$. Mean values of three independent experiments and standard deviations were shown. The Mann-Whitney Rank Sum Test was used to calculate statistical significance. An asterisk indicates a significant difference between the control group and indicated concentration groups. Statistically significant inhibition on the biofilm formation of $M$. abscessus was observed for $1 / 4 \times$ MIC, $1 / 2 \times$ MIC, MIC, and $2 \times$ MIC concentrations of C. chinensis and $1 / 2 \times$ MIC, MIC, and $2 \times$ MIC concentrations of berberine-HCl. CTL denotes control. MIC denotes minimal inhibitory concentration.

of imipenem and cefoxitin. We observed that the growth of M. abscessus was entirely inhibited by combining C. chinensis at the concentration of $2 \times \mathrm{MIC}$ with each of the 15 antibiotics tested in this study. Therefore, $C$. chinensis could significantly amplify the antibacterial effects of trimethoprim/sulfamethoxazole, clarithromycin, and linezolid against $M$. abscessus. This effect was proportional to the C. chinensis concentration.
3.4. Effect of Berberine on the MICs of Antibiotics against M. abscessus. The impact of berberine- $\mathrm{HCl}$ on the MICs of antibiotics against $M$. abscessus was also assessed. Berberine$\mathrm{HCl}$ concentrations of $1 / 4 \times \mathrm{MIC}, 1 / 2 \times \mathrm{MIC}, \mathrm{MIC}$, and $2 \times$ MIC were added to commercial 96 -well plates to measure the MICs of 15 antibiotics. Notably, the growth of M. abscessus was entirely inhibited with the addition of berberine- $\mathrm{HCl}$ at concentrations of MIC and $2 \times \mathrm{MIC}$ in the 
TABLE 1: Minimal inhibitory concentrations of antibiotics alone and in combination with Coptis chinensis in different concentrations to treat Mycobacterium abscessus.

\begin{tabular}{|c|c|c|c|c|c|}
\hline \multirow{3}{*}{ Antibiotic (target) } & \multicolumn{5}{|c|}{ Minimal inhibitory concentration $(\mu \mathrm{g} / \mathrm{mL})$} \\
\hline & \multirow{2}{*}{ Antibiotic alone } & \multicolumn{4}{|c|}{ Coptis chinensis } \\
\hline & & $1 / 4 \times \mathrm{MIC}$ & $1 / 2 \times \mathrm{MIC}$ & $\mathrm{MIC}^{\mathrm{a}}$ & $2 \times \mathrm{MIC}$ \\
\hline \multicolumn{6}{|l|}{ Folate } \\
\hline TMP/SXT & $>8 / 152$ & $>8 / 152$ & $8 / 152$ & $4 / 76$ & NG \\
\hline \multicolumn{6}{|l|}{ Protein (50S) } \\
\hline CLA & 0.5 & 0.5 & 0.25 & 0.12 & NG \\
\hline LZD & 32 & 16 & 4 & 4 & NG \\
\hline \multicolumn{6}{|l|}{ Protein (30S) } \\
\hline AMI & 16 & 16 & 16 & 16 & NG \\
\hline TOB & 16 & 16 & 16 & 16 & NG \\
\hline DOX & $>16$ & $>16$ & $>16$ & $>16$ & NG \\
\hline MIN & $>8$ & $>8$ & $>8$ & $>8$ & NG \\
\hline TGC & $0.5-1$ & 1 & 2 & 2 & NG \\
\hline \multicolumn{6}{|l|}{ Cell wall } \\
\hline IMI & 64 & 64 & 64 & 32 & NG \\
\hline FOX & 64 & 64 & 64 & $32-64^{\mathrm{b}}$ & NG \\
\hline FEP & $>32$ & $>32$ & $>32$ & $>32$ & NG \\
\hline $\mathrm{AXO}$ & $>64$ & $>64$ & $>64$ & $>64$ & NG \\
\hline AUG & $>64 / 32$ & $>64 / 32$ & $>64 / 32$ & $>64 / 32$ & NG \\
\hline \multicolumn{6}{|l|}{ Nucleic acid } \\
\hline CIP & $>4$ & $>4$ & $>4$ & $>4$ & $\mathrm{NG}$ \\
\hline MXF & $>8$ & $>8$ & $>8$ & $>8$ & NG \\
\hline
\end{tabular}

${ }^{\mathrm{a}} \mathrm{MIC}=1.5 \mathrm{mg} / \mathrm{mL}$. ${ }^{\mathrm{b}}$ One data point was measured at $64 \mu \mathrm{g} / \mathrm{ml}$. TMP/SXT, Trimethoprim/sulfamethoxazole; CLA, clarithromycin; LZD, linezolid; IMI, imipenem; FOX, cefoxitin; CIP, ciprofloxacin; MXF, moxifloxacin; DOX, doxycycline; MIN, minocycline; AMI, amikacin; TOB, tobramycin; AUG, amoxicillin/clavulanic acid; AXO, ceftriaxone; FEP, cefepime; TGC, tigecycline; NG, no growth.

15-antibiotic well plates. As shown in Table 2, berberine- $\mathrm{HCl}$ concentration at $1 / 4 \times$ MIC only reduced the MIC of trimethoprim/sulfamethoxazole. Interestingly, we observed reductions of MICs in 9 of the $15(60 \%)$ antibiotics that were tested (trimethoprim/sulfamethoxazole, clarithromycin, linezolid, amikacin, tigecycline, imipenem, cefepime, cefoxitin, and ciprofloxacin) when combined with berberine- $\mathrm{HCl}$ at a concentration of $1 / 2 \times$ MIC. Similar to the effects of $C$. chinensis, the synergistic effects of berberine- $\mathrm{HCl}$ were more significant with trimethoprim/sulfamethoxazole, clarithromycin, and linezolid. These observations indicate that berberine- $\mathrm{HCl}$ can enhance the antimycobacterial activity of most antibiotics against $M$. abscessus.

\section{Discussion}

The development of novel antimycobacterial drugs is ongoing. The antimicrobial, anti-inflammatory, and synergistic antibacterial effects of CHMs have been demonstrated in several studies $[16,20,21,30,31]$. Single extracts or formulations of CHM alone, or in combination with antibiotics, may be used to treat common bacterial infections such as MRSA, Ureaplasma urealyticum, and genital Chlamydia trachomatis infections [20,32]. In 2007, Liu et al. reported that 26 of 58 (44.8\%) CHMs used in Taiwan exhibited antibacterial activity against antibiotic-resistant Pseudomonas aeruginosa, and one of the studied herbal medicines (Ramulus Cinnamomi) could yield a synergistic effect with antibiotics [30]. In the current study, we demonstrated that C. chinensis and its major constituent, berberine, can inhibit the growth of M. abscessus. The MICs of C. chinensis and berberine- $\mathrm{HCl}$ were $1.5 \mathrm{mg} / \mathrm{mL}$ and $0.25 \mathrm{mg} / \mathrm{mL}$, respectively.

The formation of biofilm is a key mechanism in the pathogenicity and resistance of human NTM infections. Bacteria within a biofilm exhibit higher virulence to human and resistance to antimicrobials and disinfectants. Complete differentiation, achievement of maximal replication, horizontal gene exchanges and mutations, and more adaption to environment were reported [2,33]. In addition, it influences the effectiveness of endoscope and invasive device disinfection by automated processors in hospitals [34, 35]. Biofilm formation is usually an important factor in antimicrobial resistance and can lead to treatment failure. In 2011, Lin et al. reported that an active ingredient in commonly used Chinese herbal plants had inhibitory effects on S. aureus biofilm formation [36]. The capability of C. chinensis and berberine to reduce biofilm formation by M. abscessus was proven in this study. Biofilm is a complex multicellular structure that forms on bacterial surfaces. The complexity of mycobacterial biofilm, the synthesis of an extracellular matrix, usually involves various molecules including glycopeptidolipids, DNA such as groEL1 gene, and mycolic acids. The process of biofilm development is also influenced and regulated by nutrients, ions, and carbon sources [37]. Some proteins, such as adhesins, are believed to potentiate the aggregation of mycobacteria and their ability to bind to other cells [38]. Mycolic acids were reported to contribute to the resistance of mycobacteria to antibiotics and disinfectants [39]. The detailed mechanisms of reducing biofilm formation in $M$. abscessus for $C$. chinensis and berberine need further investigation. 
TABle 2: Minimal inhibitory concentrations of antibiotics alone and in combination with berberine- $\mathrm{HCl}$ at different concentrations to treat Mycobacterium abscessus.

\begin{tabular}{|c|c|c|c|c|c|}
\hline \multirow{3}{*}{$\begin{array}{l}\text { Antibiotic } \\
\text { (target) }\end{array}$} & \multicolumn{5}{|c|}{ Minimal inhibitory concentration $(\mu \mathrm{g} / \mathrm{mL})$} \\
\hline & \multirow{2}{*}{$\begin{array}{l}\text { Antibiotic } \\
\text { alone }\end{array}$} & \multicolumn{4}{|c|}{ Berberine-HCl (mg/mL) } \\
\hline & & $1 / 4 \times \mathrm{MIC}$ & $1 / 2 \times \mathrm{MIC}$ & $\mathrm{MIC}^{\mathrm{a}}$ & $2 \times \mathrm{MIC}$ \\
\hline \multicolumn{6}{|l|}{ Folate } \\
\hline $\mathrm{TMP} / \mathrm{SXT}$ & $>8 / 152$ & $8 / 152$ & $4 / 76$ & NG & NG \\
\hline \multicolumn{6}{|l|}{ Protein $(50 S)$} \\
\hline CLA & 0.5 & 0.5 & 0.12 & NG & NG \\
\hline LZD & 32 & 32 & 4 & NG & NG \\
\hline \multicolumn{6}{|l|}{ Protein $(30 S)$} \\
\hline AMI & 16 & 16 & 8 & NG & NG \\
\hline TOB & 16 & 16 & 16 & NG & NG \\
\hline DOX & $>16$ & $>16$ & $>16$ & NG & NG \\
\hline MIN & $>8$ & $>8$ & $>8$ & NG & NG \\
\hline TGC & $0.5-1$ & $0.5-1$ & $0.25-0.5^{b}$ & NG & NG \\
\hline \multicolumn{6}{|l|}{ Cell wall } \\
\hline IMI & 64 & 64 & 32 & NG & NG \\
\hline FOX & 64 & 64 & 32 & NG & NG \\
\hline FEP & $>32$ & $>32$ & 32 & NG & NG \\
\hline $\mathrm{AXO}$ & $>64$ & $>64$ & $>64$ & NG & NG \\
\hline AUG & $>64 / 32$ & $>64 / 32$ & $>64 / 32$ & NG & NG \\
\hline \multicolumn{6}{|l|}{ Nucleic acid } \\
\hline CIP & $>4$ & $>4$ & 4 & NG & NG \\
\hline MXF & $>8$ & $>8$ & $>8$ & NG & NG \\
\hline
\end{tabular}

${ }^{\mathrm{a}} \mathrm{MIC}=0.25 \mathrm{mg} / \mathrm{mL}$. ${ }^{\mathrm{b}}$ Twofold reduction of MIC for combination with berberine- $\mathrm{HCl} 0.125 \mathrm{mg} / \mathrm{mL}$. TMP/SXT, trimethoprim/sulfamethoxazole; CLA, clarithromycin; LZD, linezolid; IMI, imipenem; FOX, cefoxitin; CIP, ciprofloxacin; MXF, moxifloxacin; DOX, doxycycline; MIN, minocycline; AMI, amikacin; TOB, tobramycin; AUG, amoxicillin/clavulanic acid; AXO, ceftriaxone; FEP, cefepime; TGC, tigecycline; NG, no growth.

Antimicrobial therapies for M. abscessus infections have been investigated for decades. However, treatment regimens vary in duration, tolerability, and success. Clarithromycin, amikacin, and cefoxitin are the most commonly reported antimycobacterial choices for the treatment of M. abscessus infections [14]. Macrolide-based regimens in combination with intravenously administered antimicrobials are the currently recommended therapy [40]. However, poor responsiveness to antimicrobial chemotherapy, including macrolide-based regimens for $M$. abscessus infections, remains a challenging problem. Furthermore, side effects such as gastrointestinal upset, skin changes, and renal insufficiency are common and frequently require changing or discontinuing antibiotic therapy [14].

Previous literature has reported that combining antibiotics with CHM can produce better curative effects when treating pulmonary tuberculosis [19]. Treating intractable mycobacteriosis with antibiotics and CHMs that exhibit immunoadjunctive effects has been reported with favorable outcomes [41]. Likewise, the synergistic effects of the herbal plant extract Thymbra spicata L. and antibiotics against common, multidrug-resistant S. aureus, and Klebsiella pneumoniae strains were reported in 2016 [31]. Similar antibacterial effects of antibiotics in combination with Chinese herbal remedies for $M$. abscessus infections can be expected, but currently there are no such reports in the literature.
C. chinensis and berberine- $\mathrm{HCl}$ were proven to inhibit $M$. abscessus growth in a dose-dependent manner. Additionally, both $\mathrm{C}$. chinensis and berberine- $\mathrm{HCl}$ significantly strengthened the antimicrobial activities of trimethoprim/ sulfamethoxazole, clarithromycin, and linezolid against M. abscessus. According to measurements taken in this study, the MIC of linezolid was $32 \mu \mathrm{g} / \mathrm{mL}$, defined as resistant to antimicrobial susceptibility for $M$. abscessus. The additions of both of these CHMs reduced its MIC to $4 \mu \mathrm{g} /$ $\mathrm{mL}$, which is categorized as a susceptible level of linezolid against $M$. abscessus. Both parenteral and oral linezolid preparations are available for clinical use. The clinical implications of the combination of linezolid and C. chinensis or berberine may be prospective and therefore are recommended for long-term treatment of $M$. abscessus infections.

Although we found that berberine demonstrated an antimycobacterial effect on $M$. abscessus, the antibacterial mechanism of berberine on $M$. abscessus growth remains unknown, although previous studies have provided some useful information. Peng et al. reported that berberine could damage the cell membrane and walls of Streptococcus agalactiae, thereby increasing membrane permeability, and inhibit protein and DNA synthesis in cells [25]. Amin et al. also reported that berberine could inhibit RNA and protein synthesis in E. coli and exhibits antimicrobial effects [42]. The authors suggest that both Gram-positive and Gramnegative bacteria are susceptible to berberine. Therefore, whether berberine could inhibit the growth of M. abscessus due to membrane integrity disruption or nucleotide acid or protein synthesis inhibition needs to be investigated further.

The antimicrobial activity of trimethoprim/sulfamethoxazole is due to its interference with folate synthesis. The antimicrobial activity of clarithromycin and linezolid is due to their inhibition of protein synthesis by binding to $50 \mathrm{~S}$ subunits of bacteria [43, 44]. Therefore, the synergistic effects of both $\mathrm{C}$. chinensis and berberine- $\mathrm{HCl}$ in combination with these three antibiotics may be because of their interference with protein and RNA synthesis, as well as the cellular membrane. In addition, combining berberine- $\mathrm{HCl}$ with antibiotics could amplify the antimicrobial activities of amikacin and tigecycline through the mechanisms mentioned above. In contrast, the MIC reductions of amikacin, tigecycline, ciprofloxacin, and cefepime, measured when they were combined with $0.125 \mathrm{mg} / \mathrm{mL}(1 / 2 \times$ MIC) berberine- $\mathrm{HCl}$ were not observed with $C$. chinensis. When compared with berberine, the attenuation of antimycobacterial activity in antibiotics is hypothesized to be antagonistic to other $C$. chinensis components. Moreover, we found that C. chinensis increases the MIC of tigecycline, which acts as a protein synthesis inhibitor by binding with $30 \mathrm{~S}$ ribosomal subunits of bacteria [45]. The relationship between C. chinensis and the $30 \mathrm{~S}$ ribosomal protein subunits of bacteria needs to be investigated further. Integrating the pleomorphic effects of $C$. chinensis and its active component berberine may contribute to our knowledge of pathways and enhance the susceptibility of $M$. abscessus to multiple antibiotics. Therefore, this herb and its main component could be used and tested as an adjunct therapy for M. abscessus infections. 


\section{Conclusions}

The results of this study demonstrate that combination treatment can enhance the effects of certain antibiotics against $M$. abscessus. Chinese and Western combination treatments deserve more attention because they can be convenient and safe. The combinations of $C$. chinensis or berberine with the antibiotics identified in this study may represent a novel strategy for the treatment of patients with M. abscessus infections.

\section{Data Availability}

The data used and/or analyzed during the current study are available from the authors upon request.

\section{Conflicts of Interest}

The authors declare that there are no conflicts of interest regarding the publication of this paper.

\section{Acknowledgments}

The authors would like to thank Lih-Tsu Li and Ying-Ju Pan at the Medical Laboratory of Hsinchu Mackay Memorial Hospital and the Tamshui Branch of Mackay Memorial Hospital for their assistance in preparing reference strains. The authors also acknowledge Pei-Ju Tsai and Yi-Min Hong in the Graduate Institute of Chinese Medicine at China Medical University for helping with the experiments and data collection. The authors also thank Kaiser Pharmaceutical Co., Ltd., for providing the C. chinensis formulations for this experiment. The research and publication of this article were funded by a grant from the section of medical research of Hsinchu Mackay Memorial Hospital, Taiwan (MMH-HB-10603), and China Medical University (CMU108-S-09).

\section{References}

[1] J. O. Falkinham 3rd, "Surrounded by mycobacteria: nontuberculous mycobacteria in the human environment," Journal of Applied Microbiolgy, vol. 107, no. 2, pp. 356-367, 2009.

[2] S. Faria, I. Joao, and L. Jordao, "General overview on nontuberculous mycobacteria, biofilms, and human infection," Journal of Pathogens, vol. 2015, Article ID 809014, 10 pages, 2015.

[3] W. L. García-Jiménez, J. M. Benítez-Medina, R. Martínez et al., "Non-tuberculous mycobacteria in wild boar (Sus scrofa) from Southern Spain: epidemiological, clinical and diagnostic concerns," Transboundary and Emerging Diseases, vol. 62, no. 1, pp. 72-80, 2015.

[4] R. M. Thomson, R. Carter, C. Tolson, C. Coulter, F. Huygens, and M. Hargreaves, "Factors associated with the isolation of nontuberculous mycobacteria (NTM) from a large municipal water system in Brisbane, Australia," BMC Microbiology, vol. 13, no. 1, p. 89, 2013.

[5] S. Y. Cho, K. R. Peck, J. Kim et al., "Mycobacterium chelonae infections associated with bee venom acupuncture," Clinical Infectious Diseases, vol. 58, no. 5, pp. e110-e113, 2014.
[6] J. H. Hung, W. C. Ko, C. Y. Chen et al., "Postoperative mycobacteroides abscessus subsp. abscessus endophthalmitis: clinical analysis of 12 clustered adults and a proposed therapeutic algorithm," Journal Microbiology, Immunology and Infection, vol. 53, no. 5, pp. 766-777, 2020.

[7] A. B. Wentworth, L. A. Drage, N. L. Wengenack, J. W. Wilson, and C. M. Lohse, "Increased incidence of cutaneous nontuberculous mycobacterial infection, 1980 to 2009: a population-based study," Mayo Clinic Proceedings, vol. 88, no. 1, pp. 38-45, 2013.

[8] H. Y. Chen, C. Y. Chen, C. T. Huang et al., "Skin and softtissue infection caused by non-tuberculous mycobacteria in Taiwan, 1997-2008," Epidemiology \& Infection, vol. 139, no. 1, pp. 121-129, 2011.

[9] M. Mirsaeidi, M. Farshidpour, M. B. Allen, G. Ebrahimi, and J. O. Falkinham, "Highlight on advances in nontuberculous mycobacterial disease in North America," BioMed Research International, vol. 2014, Article ID 919474, 10 pages, 2014.

[10] T. K. Marras, D. Mendelson, A. Marchand-Austin, K. May, and F. B. Jamieson, "Pulmonary nontuberculous mycobacterial disease, Ontario, Canada, 1998-2010," Emerging Infectious Diseases, vol. 19, no. 11, pp. 1889-1891, 2013.

[11] C.-C. Lai, C.-K. Tan, C.-H. Chou et al., "Increasing incidence of nontuberculous mycobacteria, Taiwan, 2000-2008," Emerging Infectious Diseases, vol. 16, no. 2, pp. 294-296, 2010.

[12] M. Mirsaeidi, R. F. Machado, J. G. N. Garcia, and D. E. Schraufnagel, "Nontuberculous mycobacterial disease mortality in the United States, 1999-2010: a population-based comparative study," PLoS ONE, vol. 9, no. 3, p. e91879, 2014.

[13] R. Nessar, E. Cambau, J. M. Reyrat, A. Murray, and B. Gicquel, "Mycobacterium abscessus: a new antibiotic nightmare," Journal of Antimicrobial Chemotherapy, vol. 67, no. 4, pp. 810-818, 2012.

[14] S. A. Novosad, S. E. Beekmann, P. M. Polgreen, K. Mackey, K. L. Winthrop, and M. abscessus Study Team, "Treatment of mycobacterium abscessus infection," Emerging Infectious Diseases, vol. 22, no. 3, pp. 511-514, 2016.

[15] Y. J. Ryu, W.-J. Koh, and C. L. Daley, "Diagnosis and treatment of nontuberculous mycobacterial lung disease: clinicians' perspectives," Tuberculosis and Respiratory Diseases, vol. 79, no. 2, pp. 74-84, 2016.

[16] R. A. Muluye, Y. Bian, and P. N. Alemu, "Anti-inflammatory and antimicrobial effects of heat-clearing Chinese herbs: a current review," Journal of Traditional and Complementary Medicine, vol. 4, no. 2, pp. 93-98, 2014.

[17] Q. Zhao, X.-Y. Chen, and C. Martin, "Scutellaria baicalensis, the golden herb from the garden of Chinese medicinal plants," Science Bulletin, vol. 61, no. 18, pp. 1391-1398, 2016.

[18] R. Wojtyczka, A. Dziedzic, M. Kępa et al., "Berberine enhances the antibacterial activity of selected antibiotics against coagulase-negative Staphylococcus strains in vitro," Molecules, vol. 19, no. 5, pp. 6583-6596, 2014.

[19] G. Shi and L. Zhang, "Effects on type 2 diabetes complicated with pulmonary tuberculosis: regiment of insulin, isoniazid, rifampicin, pyrazinamide and ethambutol versus the regiment plus Qi-boosting and Yin-nourishing decoction of Traditional Chinese Medicine," Journal of Traditional Chinese Medicine, vol. 35, no. 3, pp. 260-265, 2015.

[20] Y. Y. Yu, H. Wang, S. W. Zhang, and B. E. Wang, "Inhibition of methicillin-resistant Staphylococcus aureus by the compound Qingre granules," Chinese Medicine Journal, vol. 123, no. 8, pp. 1017-1020, 2010.

[21] F. Lai, Y. Zhang, D. P. Xie et al., "A systematic review of rhubarb (a traditional Chinese medicine) used for the 
treatment of experimental sepsis," Evidence-Based Complementary and Alternative Medicine, vol. 2015, Article ID 131283, 12 pages, 2015.

[22] S.-J. Lin, C.-S. Chen, S.-S. Lin et al., "In vitro anti-microbial and in vivo cytokine modulating effects of different prepared Chinese herbal medicines," Food and Chemical Toxicology, vol. 44, no. 12, pp. 2078-2085, 2006.

[23] F. S. Leach, "Anti-microbial properties of Scutellaria baicalensis and Coptis chinensis, two traditional Chinese medicines," Bioscience Horizons, vol. 4, no. 2, pp. 119-127, 2011.

[24] J. Wang, L. Wang, G.-H. Lou et al., "Coptidis Rhizoma: a comprehensive review of its traditional uses, botany, phytochemistry, pharmacology and toxicology," Pharmaceutical Biology, vol. 57, no. 1, pp. 193-225, 2019.

[25] L. Peng, S. Kang, Z. Yin et al., "Antibacterial activity and mechanism of berberine against Streptococcus agalactiae," International Journal of Clinical and Experimental Pathology, vol. 8, no. 5, pp. 5217-5223, 2015.

[26] E. Desmond, Susceptibility Testing Of Mycobacteria, Nocardiae, and other Aerobic Actinomycetes, Clinical \& Laboratory Standards Institute, Wayne, PA, USA, M24 A2, 2 edition, 2011.

[27] Trek Diagnostic Systems, Broth Microdilution (Mic) Method: for Rapidly Growing Mycobacteria (Rgm), Slowly Growing Nontuberculosis Mycobacteria, Nocardia and Other Aerobic Actinomycetes: Version 1.9, pp. 1-10, Trek Diagnostic Systems, East Grinstead, UK, 2012.

[28] C. Lembke, A. Podbielski, C. Hidalgo-Grass, L. Jonas, E. Hanski, and B. Kreikemeyer, "Characterization of biofilm formation by clinically relevant serotypes of group A streptococci," Applied and Environmental Microbiology, vol. 72, no. 4, pp. 2864-2875, 2006.

[29] B. Petrini, "Mycobacterium abscessus: an emerging rapidgrowing potential pathogen. review article," American Journal of Pathology, Microbiology and Immunology, vol. 114, no. 5, pp. 319-328, 2006.

[30] C.-S. Liu, T.-M. Cham, C.-H. Yang, H.-W. Chang, C.-H. Chen, and L.-Y. Chuang, "Antibacterial properties of Chinese herbal medicines against nosocomial antibiotic resistant strains of Pseudomonas aeruginosa in Taiwan," The American Journal of Chinese Medicine, vol. 35, no. 06, pp. 1047-1060, 2007.

[31] M. F. Haroun and R. S. Al-Kayali, "Synergistic effect of Thymbra spicata L. extracts with antibiotics against multidrug-resistant Staphylococcus aureus and Klebsiella pneumoniae strains," Iranian Journal of Basic Medical Sciences, vol. 19, no. 11, pp. 1193-1200, 2016.

[32] Y. Ruan, Z. Han, and X. Han, "Report: clinical curative effect of traditional Chinese medicine combined with doxycycline in the treatment of genital Chlamydia trachomatis and urea plasma urealyticum infections," Pakistan Journal of Pharmaceutical Sciences, vol. 28, no. 4 Suppl, pp. 1555-1557, 2015.

[33] L. Hall-Stoodley and P. Stoodley, "Biofilm formation and dispersal and the transmission of human pathogens," Trends in Microbiology, vol. 13, no. 1, pp. 7-10, 2005.

[34] S. Sousa, M. Bandeira, P. A. Carvalho, A. Duarte, and L. Jordao, "Nontuberculous mycobacteria pathogenesis and biofilm assembly," International Journal of Mycobacteriology, vol. 4, no. 1, pp. 36-43, 2015.

[35] A. C. Balsamo, K. U. Graziano, R. P. Schneider, M. Antunes Junior, and R. A. Lacerda, "Remoção de biofilme em canais de endoscópios: avaliação de métodos de desinfecção atualmente utilizados," Revista da Escola de Enfermagem da USP, vol. 46, no. spe, pp. 91-98, 2012.
[36] M.-H. Lin, F.-R. Chang, M.-Y. Hua, Y.-C. Wu, and S.-T. Liu, "Inhibitory effects of 1,2,3,4,6-penta-O-Galloyl- $\beta$-d-Glucopyranose on biofilm formation by Staphylococcus aureus," Antimicrobial Agents and Chemotherapy, vol. 55, no. 3, pp. 1021-1027, 2011.

[37] J. Esteban and M. Garcia-Coca, "Mycobacterium biofilms," Frontiers in Microbiology, vol. 8, p. 2651, 2017.

[38] F. D. Menozzi, J. H. Rouse, M. Alavi et al., "Identification of a heparin-binding hemagglutinin present in mycobacteria," Journal of Experimental Medicine, vol. 184, no. 3, pp. 9931001, 1996.

[39] M. M. Zambrano and R. Kolter, "Mycobacterial biofilms: a greasy way to hold it together," Cell, vol. 123, no. 5, pp. 762-764, 2005.

[40] M. R. Lee, W. H. Sheng, C. C. Hung, C. J. Yu, L. N. Lee, and P. R. Hsueh, "Mycobacterium abscessus complex infections in humans," Emerging Infectious Diseases, vol. 21, no. 9, pp. 1638-1646, 2015.

[41] H. Tomioka, "Usefulness of Chinese herbal medicines as hostdirected therapeutics against mycobacterial infections: a review," The American Journal of Chinese Medicine, vol. 45, no. 08 , pp. 1597-1611, 2017.

[42] A. H. Amin, T. V. Subbaiah, and K. M. Abbasi, "Berberine sulfate: antimicrobial activity, bioassay, and mode of action," Canadian Journal of Microbiology, vol. 15, no. 9, pp. 10671076, 1969.

[43] D. P. Sangurdekar, Z. Zhang, and A. B. Khodursky, "The association of DNA damage response and nucleotide level modulation with the antibacterial mechanism of the antifolate drug trimethoprim," BMC Genomics, vol. 12, p. 583, 2011.

[44] M. A. Kohanski, D. J. Dwyer, and J. J. Collins, "How antibiotics kill bacteria: from targets to networks," Nature Reviews Microbiology, vol. 8, no. 6, pp. 423-435, 2010.

[45] M. Bhattacharya, A. Parakh, and M. Narang, "Tigecycline," Journal of Postgraduate Medicine, vol. 55, no. 1, pp. 65-68, 2009. 